\title{
COMPUTER GUIDED MAXILLARY POSITIONING USING OSTEOTOMY/PLATES LOCATING SURGICAL GUIDES IN MANAGEMENT OF MAXILLARY DEFICIENCY
}

\author{
Niveen Askar* and Mohammed Omara**
}

\begin{abstract}
Maxillary segment repositioning is essential for esthetic and functional outcomes. Increased success and experience achieved with computer guided Le Fort I osteotomy turned the attention to develop surgical techniques to cope with deficient maxilla solving the problem of the timeconsuming manual steps of intermediate wafer fabrication in addition to its inability to adjust the correct vertical position of the maxilla in relation to the skull base. This study aimed to evaluate $\mathrm{CAD} / \mathrm{CAM}$ generated osteotomy/ plates locating surgical guides in three-dimensional maxillary segment repositioning. Ten patients with maxillary hypoplasia were subjected to virtual model 3D skull models for titanium plates pre-bending. Screw holes were surgery forming corrected 3D skull models for construction of osteotomy / plates locating surgical marked to the corrected guides. Pre-bent plates were installed intra-operatively into the predetermined screw holes. Placement of the plates adjust the depressed maxilla three dimensionally. All patients were clinically and radiographically evaluated pre and post-operatively. Degree of three-dimensional corrective movement were measured and statistically evaluated. CAD/CAM generated osteotomy plates locating surgical guides were proved to be more reliable for three-dimensional maxillary .segment repositioning
\end{abstract}

KEY WORDS: Computer guided, Maxillary repositioning, Orthognathic Surgery

\section{INTRODUCTION}

In corrective jaw surgery, maxillary segment repositioning after Le Fort I osteotomy is a fundamental surgical step to achieve the best postoperative facial esthetics and functional outcomes. In conventional articulator model surgery, the intermediate wafer was the mainly utilized essential tool for intra-operative maxillary repositioning. ${ }^{1-4}$ The pre-operative work-up procedure for fabricating intermediate wafers requires several manual steps that can be time-consuming and may induce technical errors., ${ }^{5,6}$ In addition, the correct intraoperative vertical maxillary position in relation

\footnotetext{
* Associate Professor at department of Oral \& Maxillofacial surgery, Faculty of Dentistry,Cairo University,Egypt

** Lecturer at the department of oral and maxillofacial surgery. Affiliation: Faculty of oral and dental medicine, Cairo University, Egypt
} 
to the skull base must be manually adjusted by the operator as the intermediate wafer only guarantee the transverse and sagittal repositioning. Moreover, the accuracy of intra-operative titanium plates bending is questionable and can be time-consuming. , 2,7-9 $^{2}$

The revolution of the new technologies available for preoperative planning, simulation, creation of patient-specific models, and intraoperative guidance are becoming more user-friendly and attractive to a broader field of orthognathic surgeons ${ }^{10}$. CAD/ CAM technologies have been utilized to generate intermediate

surgical wafers to transfer the virtual measures of the computer simulation to the actual operating surgical theater. ${ }^{11-13}$ Technical errors during surgical wafer fabrication can be reduced with accurate simulation, but vertical maxillary repositioning remains non-guaranteed surgical step that depends on the conventional surgical maneuvers operated by the surgeon. Precise positioning of the maxilla is not easy to achieve especially when complex threedimensional movements are needed ${ }^{8}$

Authors adopted the use of bone borne surface templates to be an alternative to the use of an intermediate wafer. Three-dimensional movement and maxillary repositioning were achieved guided by bone borne surface templates and maxillary segment was aligned to the skull base rather than using the wafer and depending on the mandible. ${ }^{14-23}$ The current study aimed to evaluate CAD/CAM generated osteotomy/ plates locating surgical guides in three-dimensional maxillary segment repositioning.

\section{PATIENTS AND METHODS}

A total of ten consecutive patients with maxillary deficiency were selected from the outpatient clinics of Oral and Maxillofacial Surgery Department and Orthodontic Department, Faculty of Oral and Dental Medicine, Cairo University. All patients were nonsyndromic suffering from maxillary deficiency associated with occlusal discrepancies. Patients with any systemic disease, intra-bony lesions or infections were excluded from the study.

A thorough preoperative assessment of all patients was carried out including history taking, clinical, photographic (Canon Camera 6d full frame, Canon lens 100 macro I, Ring box lighting, Japan) and radiographic examinations in addition to dental casts analysis. ${ }^{29} \mathrm{~A}$ panoramic radiography and lateral cephalometry (OT100 Instrumentarium imaging Finland $16 \mathrm{~mA} 85 \mathrm{KV}$ ) were requested from all patients for preliminary survey and initial assessment utilizing computer software (Onyx ceph 2.6.24: image instrument, $\mathrm{GmbH}$ ).

\section{Preoperative work-up}

Dental casts were mounted in a simple hinge articulator in both preoperative and preplanned post-operative occlusion by the patient's orthodontist. Maxillary acrylic vacuum formed stent (1mm thickness) was fabricated in the centric relation of the patient and five carbide burs size 2 were inserted in the acrylic during its setting at the following areas: (Mid-line, bilateral canine's areas $\&$ bilateral molar's areas) representing reference points or registration markers forming what is called registration wafer. The dental casts together with the registration wafer were laser scanned separately and in a preoperative and postulated postoperative occlusion forming STL files.

Preoperative Computed Tomography (CT) of the head and neck region was acquired utilizing multislice CT machine (Light speed plus, general electric healthcare-USA) according to the following protocol (Axial slices thickness: $1 \mathrm{~mm}$ thick or thinner, Distance between slices: $1 \mathrm{~mm}$ or less, Gantry should not be tilted (gantry tilt $=0$ ), All axial cuts in DICOM format were recorded, FOV should be limited, but all the region of interest described in the request should be included. The patient's mouth should be kept open during CT scanning to ensure 
the separation between mandible and maxilla utilizing the same previously mentioned registration wafer with its registration markers (carbide burs heads), Voxel ratio should be 1:1 (pitch =1:1), The window should be standard, in the original format of the CT scanner).

CT scan with DICOM format was imported into specialized surgical simulation software (mimics) (Mimics: Materialize, Belgium) to generate virtual skull model. Digital casts obtained from 3D scanning of plaster dental casts were imported and registered over the dental arches of the virtual 3D skull model forming what is called composite skull model. The registration procedures were guided by matching the registration markers (carbide burs heads) inserted in the registration wafer which was laser scanned at the first time while dental casts occluded on it, and CT scanned another time while patient biting on it.

The virtual composite skull model was viewed in multiplanar view (axial, coronal, sagittal and 3D views). Three-dimensional (3D) analysis using the built-in reference points and planes from the measures and analysis in the drop-down menu of the simulation icon of the tool bar of the mimic program together with the clinical examination were performed to accurately quantify the degree of deformity and determine the osteotomy level, configuration and the degree of the required 3D movement.

Segmentation process was carried out while orientation of the 3D skull model depending on Frankfort horizontal, mid-sagittal and coronal planes. A cut plane was created unilaterally in only one side of the composite skull model to simulate the designed osteotomy line which was extended in the form of classical Le fort I osteotomy. Based on the concept of computerized mirror imaging the osteotomy configuration was mirror imaged to the other side to guarantee a symmetrical osteotomy regarding leveling and configuration. (Fig. 1A)

The osteotomized segment was repositioned according to the treatment plan generated from the $3 \mathrm{D}$ analysis of the composite skull model together with data obtained from the clinical examination. Repositioning of the maxillary segment to the postoperative position was done by creating a duplicate of the maxillary segment below the designed osteotomy cut plane, followed by movement of the duplicate element in 3D planes: mediolaterally for roll adjustment to correct any mid-line deviation, Vertically for Yaw adjustment and antro-posteriorly for Pitch adjustment. This process is called repointion. (Fig. 1B) After repointion was done, a soft tissue thresholding was performed to arbitrary predict the post-operative soft tissue outcome.

After final positioning of the osteotomized maxillary segment. Surface templates were designed above and below the designed osteotomy line. (Fig. 1C) The finally adjusted post-operative bony segment position with the rest of the model and surface templates were exported from the mimic's software to be imported in CAD software (3D max 2009, autodeskinc-San Rafael,CA).

The imported CAD software files were exported in the form of sterolithographic format to a multijet modelling (thermal material application with ultra-violet curing) 3D printing machine (InVision Si2,3Dsystems - Rock Hill, SC.) to produce the steriolithographic model and surface templates. Both model and templates were formed from a plastic material (VisiJet SR200,3D Systems - Rock Hill, SC.).

Conventional 2.0 titanium mini-plates were selected and perfectly adapted over the surgically corrected and printed 3D skull model. The prebent mini plates were fixed in its place on the 3D skull model through drilling of the model and screw fixation. (Fig. 2A) Screw holes marked on the 3D skull model after pre-bent plate removal were through and through further drilled. Printed surface templates were adapted over the 3D skull model followed by reverse drilling of the surface 

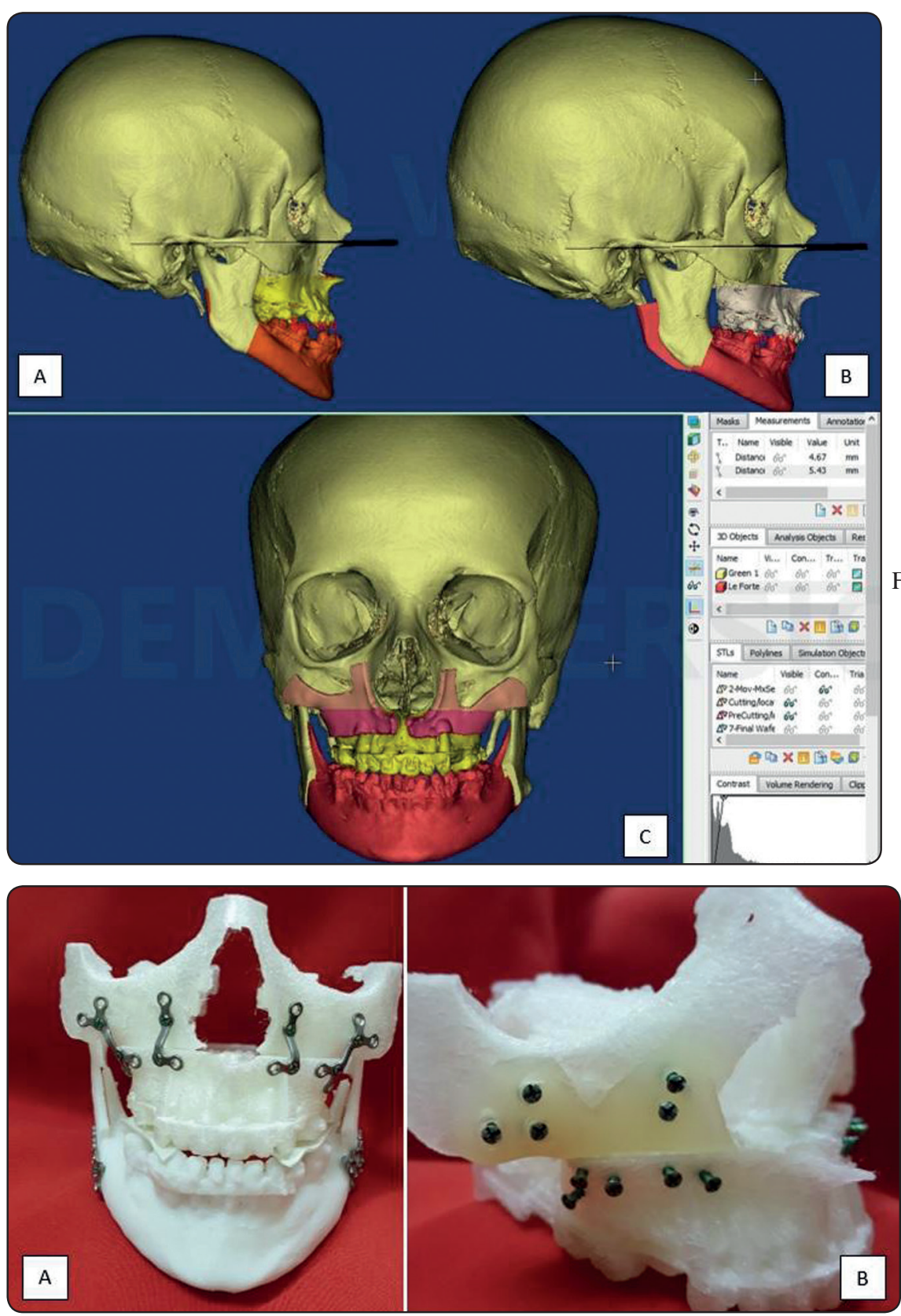

Fig. (1) Snapshots of 3D skull model window in mimics program showing: (A) Virtual cutting plane. (B) Virtual segmentation and 3D simulation to the desired post-operative maxillary position. (C) Virtual fabrication of osteotomy/plates surgical guides.

Fig. (2) A clinical photograph showing (A) 3D printed skull model with pre-bended and fixed titanium mini plates. (B) Osteotomy/screw holes locating surgical guides adapted over the 3D printed skull model after reverse drilling of the locating holes. templates forming surgical guides of dual use: osteotomy guidance and plates' screw holes location (osteotomy / plates locating surgical guides) (Fig. 2B)

The end products of the pervious computergenerated work-up were the pre-bent plates and the osteotomy / plates locating surgical guides. Both together were only utilized to transfer and execute the treatment plan.

\section{Surgical procedures}

All surgeries were operated at the Faculty of Oral and Dental Medicine, Cairo University after patients signed on an informed consent including all the complications of the surgical procedures. The procedures were performed under general anaesthesia with nasotracheal intubation and controlled hypotension. Local anesthesia utilized was Mepivicaine Hydrochloride with 1:200,000 of Adrenaline solution (Scandonest 2\% by Septodent, France.) 
for hemostasis. Scrubbing of the patients was performed utilizing betadine (Povidone iodine) surgical scrub. Draping of the patients was carried out in a standard fashion.

Intraoral mandibular incisions were performed bilaterally and reflected exposing the bone for bilateral sagittal split osteotomy (BSSO) after performing the cuts and before bone splitting an intraoral vestibular maxillary incision was performed and reflected exposing the anterior-lateral walls of the maxilla. The dissection was extended as laterally as possible to expose the postro-lateral aspect of the maxilla.

After wide exposure of the deficient maxillary region, four osteotomy / plates locating surgical guides were anatomically adapted and fixed using screws over the exposed surface of the facial skeleton. Two guides were placed per each side of the face. Two guides were located superior to the others leaving a slit. The created slit delineated the exact osteotomy level and configuration. The holes marked on the surgical guides that guarantee the exact plates' holes location were drilled prior to the osteotomy.

Le Fort I osteotomy was then performed with a reciprocating saw guided by the slit that created by the previously adaptation of the surgical guides, starting from the lateral aspect of the pyri-form aperture and extended laterally to the pterygoids. This osteotomy was completed using spatula chisels. The osteotomy of the medial walls of the maxillary sinuses was carried out in a higher position. Particular attention must be drawn to pterygomaxillary osteotomy both apically and medially in order to simplify the mobilization of the maxillary segment.

After osteotomy, the osteotomized segment was down fractured while the osteotomy / plates locating surgical guides fixed in place to protect the bone during the segment manipulation. Finally, the surgical guides were removed and the pre-bent plates were installed by placing screws in the predetermined screw holes. Plates installation adjust the osteotomized maxillary segment to the pre-planned position three dimensionally. (Fig. 3A-C)

Finally, Alar base was sutured using Cinch technique utilizing 2,0 proline sutures. Then the vestibular incision was closed in a V\&Y suturing technique utilizing a 3,0 resorbable sutures. (Polyglycoic acid coated braided suture, CFIRM esnetKratznedelfabrik, Germany.)

\section{Postoperative evaluation}

Postoperative follow up was carried out every other week for the first month, then, every month for 3 months to evaluate the wound healing and edema resolution. Postoperative panoramic radiographs were performed one-week postoperatively for overall screening of the plates and screws. Multi-slice CT scan was performed one month postoperatively to be compared with preoperative $\mathrm{CT}$ and the preoperative corrected virtual skull model.

Assessment of the technical accuracy of $3 D$ maxillary segment repositioning (Virtually corrected versus actual post-operative $3 D$ skull models) according to the following protocol (Fig. 4):

1. Identification of the built-in reference points and planes from the measures and analysis in the drop-down menu of the simulation icon of the mimic's program tool bar.

2. Marking five reproducible bony reference points (anterior nasal spine, bilateral pyriform, bilateral greater palatine foramina) and four reproducible dental reference points (bilateral canines tips and bilateral mesio-buccal cusp tips of maxillary first molars).

3. Measuring the distances from these points to the constructed coronal, Frankfort horizontal and mid sagittal planes respectively and taking the average of these distances for each patient's virtually corrected versus actual postoperative 3D skull models to be compared for assessment of the sagittal, vertical and transverse movements of the maxillary segment respectively. 

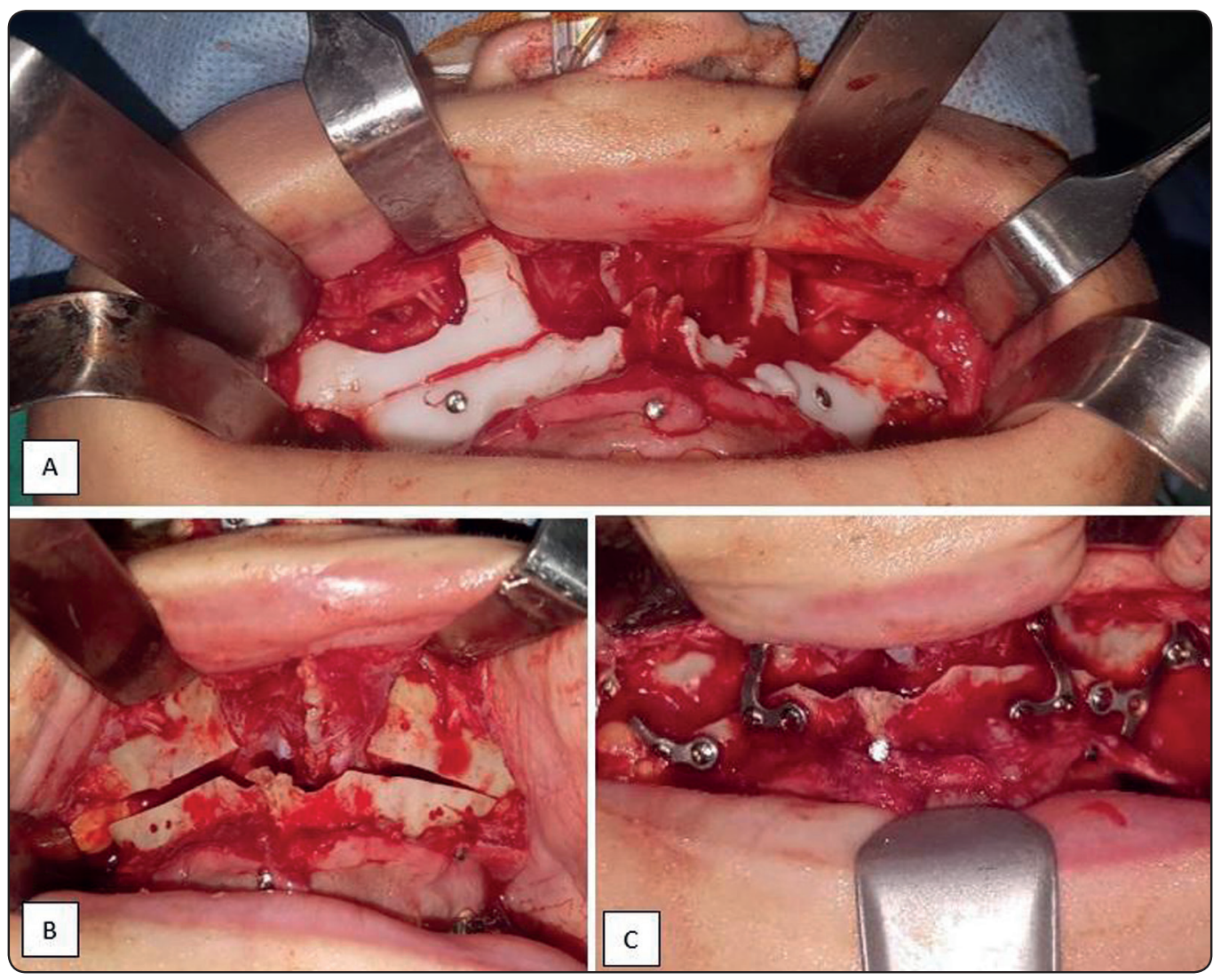

Fig. (3) Intra-operative clinical photograph showing: (A) Osteotomy/plates locating surgical guides anatomically adapted on the facial skeleton. (B) Clean cut osteotomy and screw holes for pre-bent plates location. (C) Accurate intra-operative maxillary repositioning and plates adaptation.

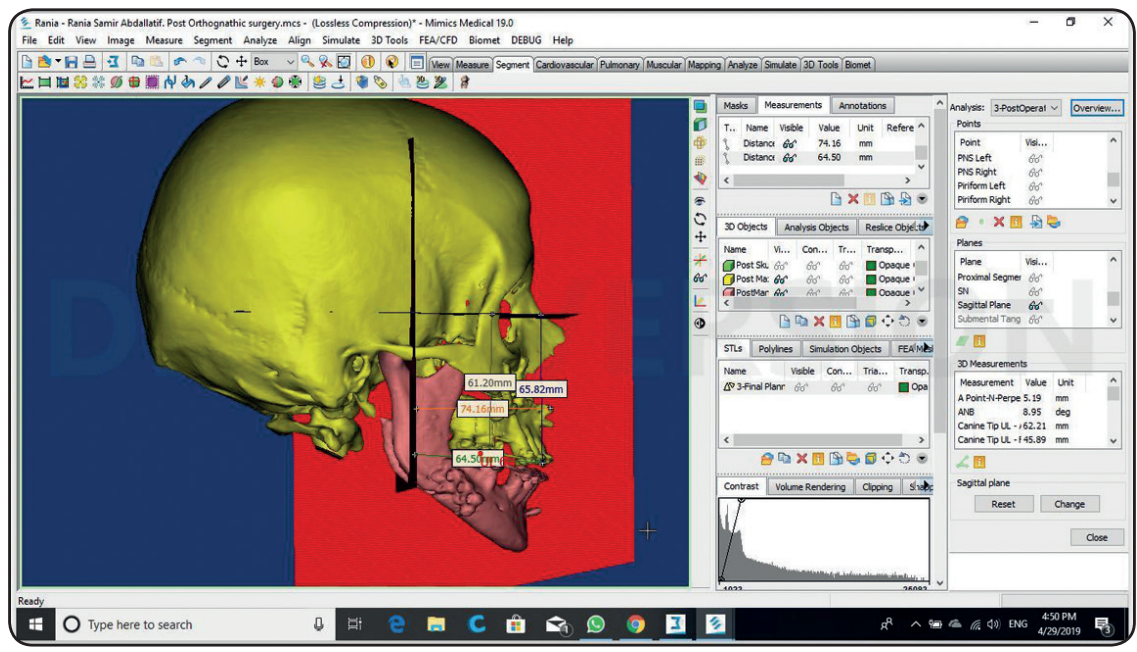

Fig. (4) A snapshot of 3D Skull model window in mimics program showing the measured bony and dental reference points in relation to the coronal, Frankfort horizontal and sagittal planes plane. 


\section{Statistical Analysis:}

All data were subjected to statistical analysis. The statistical analysis was performed using IBM SPSS version 25.0 software (IBM Corp., Armonk, NY, USA). Data were represented as the mean \pm standard deviation (SD). The one-sample Kolmogorov-Smirnov test was used to examine the normality of the data distribution. The pairedsample t-test was used to compare scale data within the studied group of patients.

\section{RESULTS}

In this prospective study, a total of ten patients ( 3 males and 7 females) with an average age of 20 years (range from $18-21$ years). All patients were selected with maxillary deficiency with occlusal discrepancy. The selected patients were having non-syndromic facial skeletal deformities. All patients were allocated consecutively for computer aided maxillary repositioning. all patients were evaluated clinically and radiographically utilizing computed tomography. All patients were included for statistical analysis.

\section{Intra-operative clinical findings:}

All cases were run uneventful. Operation time was of average 5 hours per-bi-maxillary operation. All cases showed accurate bone to surgical guides adaptation. Surgical guides provided easy and accurate transfer of the pre-planned osteotomy lines and plates location in addition to its third indirect action of thin facial bone protection during down fracture of the osteotomized bone segment in all cases. Computer aided plate pre-bending and computer guided plates' screw holes drilling allow rapid and perfect plates adaptation in all cases which was positively reflected in the operation time and post-operative radiographic accuracy.
In the current study the Le Fort I osteotomy was performed in all cases without any complications and the maxillary segment was exactly positioned similar to the preoperative plan simulation that was positively reflected to the post-operative radiographic accuracy. Bi-maxillary surgical correction was performed in all cases with accurate post-operative occlusion.

\section{Post-operative clinical findings:}

The follow up period of the cases showed uneventful healing with no wound dehiscence, no plate exposure or infection. Edema resolved in all cases within 1 week. Comparative evaluation between the pre and postoperative photographs of all cases showed an esthetically acceptable appearance and post-operative occlusion. (Fig. 5)

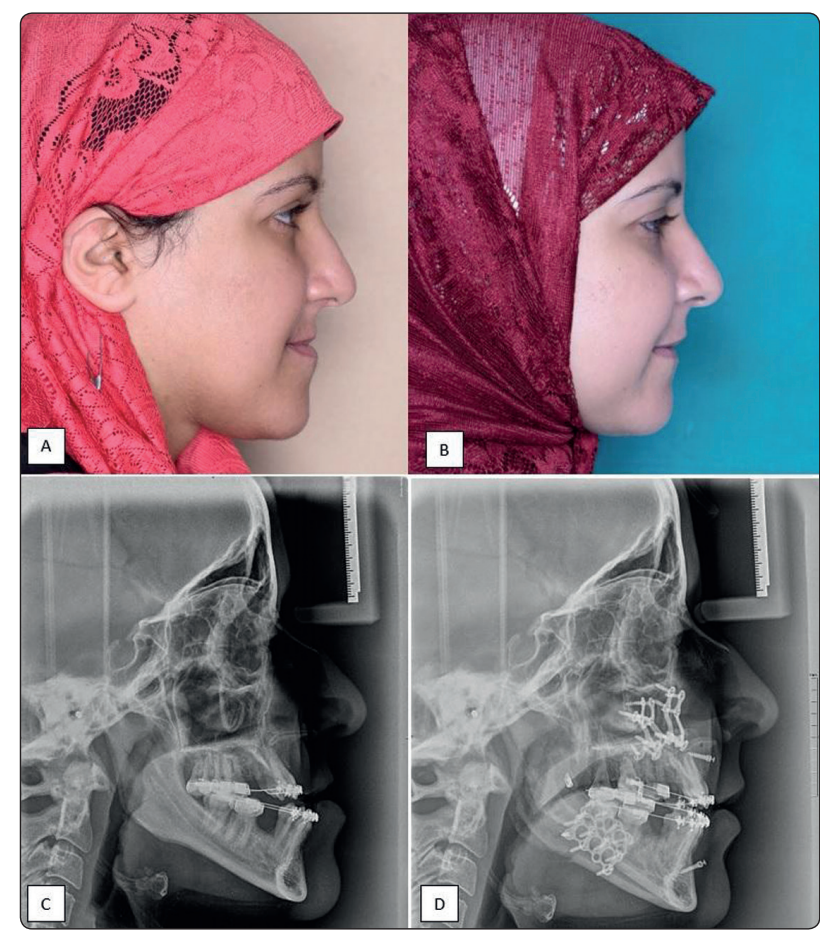

Fig. (5) A clinical photograph showing the pre-operative versus post-operative: (A and B) Facial appearance. (C and D) Lateral cephalometry. 


\section{Radiographic findings}

\section{Sagittal Maxillary segment movement in relation to the coronal plane:}

The difference between the sagittal maxillary movement regarding the dental reference points (virtual versus actual post-operative 3D skull models) was not statistically significant with mean difference $=0.025(\mathrm{p}$ value $=0.170) .($ Fig. $7 \mathrm{~A})$ (Table 1).

The difference between the sagittal maxillary movement regarding the bony reference points (virtual versus actual post-operative 3D skull models) was not statistically significant with mean difference $=0.002(p$ value $=0.805) .($ Fig. $7 B)($ Table 1$)$.

TABLE (1) Showing the descriptive Statistical difference between the sagittal maxillary movement regarding the dental and bony reference points in relation to the coronal plane (virtual versus actual post-operative 3D skull models)

\begin{tabular}{|l|c|c|}
\hline The statistical difference & Mean & P value \\
\hline Dental Measures & 0.025 & 0.170 \\
\hline Bony measures & 0.002 & 0.805 \\
\hline
\end{tabular}

Vertical Maxillary segment movement in relation to the Frankfort horizontal plane:

The difference between the vertical maxillary movement regarding the dental reference points (virtual versus actual post-operative 3D skull models) was not statistically significant with mean difference $=0.000(\mathrm{p}$ value $=1.0) .($ Fig. 7A $)($ Table 2$)$.

The difference between the vertical maxillary movement regarding the bony reference points (virtual versus actual post-operative 3D skull models) was not statistically significant with mean difference $=0.001(p$ value $=0.726) .($ Fig. $7 B)($ Table 2$)$.
TABLE (2) Showing the descriptive Statistical difference between the vertical maxillary movement regarding the dental and Bony reference points in relation to the Frankfort horizontal plane (virtual versus actual post-operative 3D skull models)

\begin{tabular}{|l|c|c|}
\hline The statistical difference & Mean & P value \\
\hline Dental Measures & 0.00 & 1.0 \\
\hline Bony measures & 0.001 & 0.726 \\
\hline
\end{tabular}

\section{Transverse Maxillary segment movement in re- lation to the sagittal plane:}

The difference between the transverse maxillary movement regarding the dental reference points (virtual versus actual post-operative 3D skull models) was not statistically significant with mean difference $=0.03(p$ value $=0.437)$. $($ Fig. $7 \mathrm{~A})$ (Table 3).

TABLE (3) Showing the descriptive Statistical difference between the transverse maxillary movement regarding the dental and bony reference points in relation to the sagital plane (virtual versus actual post-operative 3D skull models)

\begin{tabular}{|l|c|c|}
\hline The statistical difference & Mean & P value \\
\hline dental Measures & 0.03 & 0.437 \\
\hline Bony measures & 0.001 & 0.726 \\
\hline
\end{tabular}

The difference between the transverse maxillary movement regarding the bony reference points (virtual versus actual post-operative 3D skull models) was not statistically significant with mean difference $=0.001(p$ value $=0.726) .($ Fig. $7 B)($ Table 3$)$. 


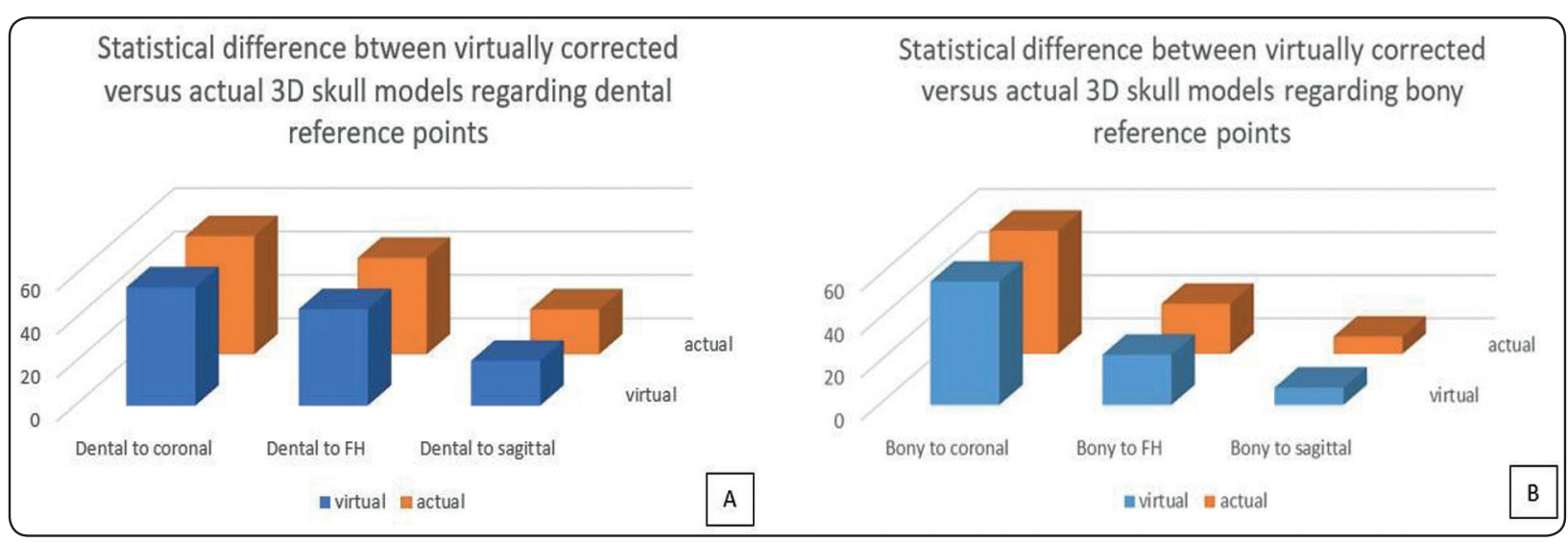

Fig. (6) (A and B) A Statistical graphs showing: The non-significant statistical difference between virtually corrected and actual postoperative 3D skull models

\section{DISCUSSION}

Conventional articulator model surgery has been utilized extensively for inter- mediate wafer fabrication in order to adjust sagittal and transverse maxillary segment position. However, its manual fabrication is time-consuming and may induces technical errors. Intra-operative maxillary segment repositioning depending on either conventional or $\mathrm{CAD} / \mathrm{CAM}$ generated intermediate wafer can be critical and difficult especially in vertical maxillary repositioning. ${ }^{4,9,11-13}$

In the current study the followed computer guided protocol was relied on the mimic simulation software program in 3D cephalometric simulation and virtual planning owing to its accuracy, simplicity and wide spreading applications in all fields of oral and maxillofacial surgeries. Virtual simulation in the current study gives a full picture about the 3D multiplanar movement of the osteotomized segment and its relation to the base of the skull and occlusion which was reflected in the net results of the study.

Based on the literature' several authors fabricated surface templates to guide the osteotomy and screw holes location. ${ }^{14-23}$ The followed computer guided protocol in the current study solved the problem of different rendering of CT scan in both dentition and facial bone. This was attained through the fabrication of registration wafer which was modified from the work of Gateno et al ${ }^{24}$.

The followed steps in the current computer guided protocol involved the essential fabrication of skull model which included both the scanned casts and the facial bone forming what's called a composite skull model that gave the chance for the fabrication of versatile guiding tools as osteotomy / plates locating surgical guides based on the work of Bay SH et al, but in a simpler, time saving and affordable manner ${ }^{15}$.

In the current study, osteotomy lines guided by the templates guaranteed accurate separation of the maxillary segment similar to that of the virtual surgical segmentation and simulation procedures. The guided pre-bent plates installation after osteotomy guaranteed precise and easily applicable three dimensional maxillary segment repositioning to the virtually preplanned position without an intermediate wafer or intraoperative measurements.

Finally, the idea of rapid prototyping (3D printing) of the virtual model in the current study with subsequent plate pre-bending and modification of the surgical guides with locating screw holes significantly reduced the operative time and 
guaranteed accurate positioning of the osteotomized segment three dimensionally.

From the results of the current study, it could be concluded that computer guided surgery was proved to be more reliable technique for three-dimensional maxillary repositioning. The preoperatively planned 3D movement of the osteotomized segment accurately transferred through the use of bone borne osteotomy / plates locating surgical guides and prebent plates. The use of computer guided model surgery simplified the operation and reduced the time of surgery, which in turn leaded to reduced blood loss and post-operative edema.

\section{REFERENCES}

1. Turvey TA: Simultaneous mobilization of the maxilla and mandible: Surgical technique and results. J Oral Maxillofac Surg 1982: 40:96.

2. Ferguson JW, Luyk NH: Control of vertical dimension during maxillary orthognathic surgery. A clinical trial comparing internal and external fixed reference points. J Craniomaxillofac Surg 1992: 20:333.

3. Ellis E III: Bimaxillary surgery using an intermediate splint to position the maxilla. J Oral Maxillofac Surg 1999: 57:53.

4. Ong TK, Banks RJ, Hildreth AJ: Surgical accuracy in Le Fort I maxillary osteotomies. Br J Oral Maxillofac Surg 2001: 39:96.

5. Baek SH, Bell WH, Chu S, et al: Distraction Osteogenesis of the Facial Skeleton. Hamilton, BC Decker 2007: p 115

6. Olszewski R: Re: How accurate is model planning for orthognathic surgery? Sharifi A, Jones R, Ayoub A, Moos K, Walker F, Khambay B, McHugh S. Int J Oral Maxillofac Surg 2008: 37:1089-1093. Int J Oral Maxillofac Surg 2009: 38:1009.

7. Kahnberg KE, Sunzel B, Astrand P: Planning and control of vertical dimension in Le Fort I osteotomies. J Craniomaxillofac Surg 1990: 18:267.

8. Schneider M, Tzscharnke O, Pilling E, et al: Comparison of the predicted surgical results following virtual planning with those actually achieved following bimaxillary operation of Dysgnathia. J Craniomaxillofac Surg 2005: 33:8.
9. Gil JN, Claus JD, Manfro R, et al: Predictability of maxillary repositioning during bimaxillary surgery: Accuracy of a new technique. Int J Oral Maxillofac Surg 2007: 36:296.

10. Rudman k, Hoekzema C., and Rhee j. Computer -assissted innovations in craniofacial surgery. J facial Plastic Surgery.2011: 27,4, 358-365.

11. Xia JJ, Gateno J, Teichgraeber JF: Three-dimensional computer aided surgical simulation for maxillofacial surgery. Atlas Oral Maxillofac Surg Clin North Am 2005: 13:25.

12. Metzger MC, Hohlweg-Majert B, Schwarz U, et al: Manufacturing splints for orthognathic surgery using a threedimensional printer. Oral Surg Oral Med Oral Pathol Oral Radiol Endod 2008:105:e1.

13. Song KG, Baek SH: Comparison of the accuracy of the three dimensional virtual method and the conventional manual method for model surgery and intermediate wafer fabrication. Oral Surg Oral Med Oral Pathol Oral Radiol Endod 2009: 107:13.

14. Bai SZ, Bo B, Bi Y, et al: CAD/CAM surface templates as an alternative to the intermediate wafer in orthognathic surgery. Oral Surg Oral Med Oral Pathol Oral Radiol Endod 2010: 110:e1.

15. Bai SH, Liu Y, Zhao J, Zhao Y. Computer-Aided Design and ComputerAided Manufacturing Locating Guides Accompanied with Prebent Titanium Plates in Orthognathic Surgery. J Oral Maxillofac Surg. 2012: 70: 2419-2426.

16. Sadiq Z, Collyer J, Sneddon K, Walsh S. Orthognathic treatment of asymmetry: two cases of "waferless" stereotactic maxillary positioning.Br J Oral Maxillofac Surg. 2012: Mar;50(2):e27-9.

17. Zinser MJ, Mischkowski RA, Dreiseidler T, Thamm OC, Rothamel D, Zöller JE. Computer-assisted orthognathic surgery: waferless maxillary positioning, versatility, and accuracy of an image-guided visualisation display.Br $\mathrm{J}$ Oral Maxillofac Surg. 2013: Dec;51(8):827-33.

18. Kwon TG, Choi JW, Kyung HM, Park HS. Accuracy of maxillary repositioning in two-jaw surgery with conventional articulator model surgery versus virtual model surgery.Int J Oral Maxillofac Surg. 2014 Jun;43(6):732-8.

19. Zhang N, Liu S, Hu Z, Hu J, Zhu S, Li Y. Accuracy of virtual surgical planning in two-jaw orthognathic surgery: comparison of planned and actual results. Oral Surg Oral Med Oral Pathol Oral Radiol. 2016: Aug;122(2):143-51.

20. Han JJ, Yang HJ, Hwang SJ. Repositioning of the Maxil- 
lomandibular Complex Using Maxillary Template Adjusted Only by Maxillary Surface Configuration Without an Intermediate Splint in Orthognathic Surgery.J Craniofac Surg. 2016: Sep;27(6):1550-3.

21. Kraeima J, Jansma J, Schepers RH. Splintless surgery: does patient-specific CAD-CAM osteosynthesis improve accuracy of Le Fort I osteotomy? Br J Oral Maxillofac Surg. 2016: Dec;54(10):1085-1089.

22. Lin X, Li B, Wang X, Shen SGF. Accuracy of maxillary repositioning by computer-aided orthognathic surgery in patients with normal temporomandibular joints. Br J Oral Maxillofac Surg. 2017: Jun;55(5):504-509.
23. Heufelder M, Wilde F, Pietzka S, Mascha F, Winter K, Schramm A, Rana M. Clinical accuracy of waferless maxillary positioning using customized surgical guides and patient specific osteosynthesis in bimaxillary orthognathic surgery. J Craniomaxillofac Surg. 2017: Sep;45(9):15781585 .

24. Gateno J, Xia JJ, Teichgraeber JF, Christensen AM, Lemoine JJ, Liebschner MAK, Gliddon MJ, Briggs ME. Clinical Feasibility of Computer-Aided Surgical Simulation (CASS) in the Treatment of Complex CranioMaxillofacial Deformities. J Oral Maxillofac Surg. 2007: 65:728-734. 